\title{
The Gaia mission \\ - a rich resource for outreach activities
}

\author{
K. S. O'Flaherty, J. Douglas and T. Prusti \\ Research \& Scientific Support Department, Directorate of the Scientific Programme, \\ European Space Agency \\ email: koflaher@rssd.esa.int; jdouglas@rssd.esa.int; tprusti@rssd.esa.int
}

\begin{abstract}
Space science missions, and astronomy missions in particular, capture the public imagination at all levels. ESA's Gaia mission is no exception to this. In addition to its key scientific goal of providing new insight into the origin, formation, and evolution of the Milky Way, Gaia also touches on many other scientific topics of broad appeal, for example, solar system objects, stars (including rare and exotic ones), dark matter, gravitational light bending. The mission naturally provides a rich resource for outreach possibilities whether it be to the general public, or to specific interest groups, such as scientists from other fields or educators. We present some examples of possible outreach activities for Gaia.
\end{abstract}

Keywords. astrometry, sociology of astronomy, education, space vehicles

\section{Introduction}

A number of key papers describing the science of Gaia are included in these proceedings and we will not elaborate on this area except to note that the key science questions addressed by Gaia are of immediate and apparent appeal to many diverse audiences. Gaia is one of Europe's flagship astronomy missions of the next decade. All parties involved have a responsibility to contribute to, or support, outreach activities aimed at promoting the mission, and its discoveries, to the widest audience possible.

What is meant by outreach? Here we consider outreach activities in the broadest of terms: those which go beyond our normal scope of activity to provide information, resources or services to audiences not in our immediate service area. The goals of astronomy outreach include: connecting the public to the wonder and excitement of astronomy, cultivating an interest and appreciation of science in students (of all ages), sharing results and discoveries with colleagues, peers and interested parties. The actors who participate, and their roles, are varied. ESA, national funding agencies, scientific institutes, and individual astronomers will all participate in Gaia outreach. Each of these groups has their own constituency, motivation, speciality and body of resources. This will determine the activities in which they participate, and the timing and content of their outreach contributions. To be effective, outreach activities need to be focussed to the interests, expectations and delivery means appropriate for each audience.

One of the immediate challenges for Gaia will be how to represent the results. The problem is encapsulated in this observation: "Beautiful colour images of the sky are both a blessing and a curse for the communication of astronomy to the public. While undoubtably attractive they can obscure the fact that discoveries are often made in astrophysics using techniques and measurements that are much more difficult to grasp and certainly less appealing to view." (Fosbury, 2005). The Hubble era has led audiences to expect stunning 'photographs' of the Universe. Careful consideration of how to represent and visualise the Gaia results will be required to respond to audience expectations. 


\section{Outreach activities for Gaia: some examples}

\subsection{Outreach to school children}

The school-going population of today, students aged 5 to 18 years, are the scientists and software engineers of the Gaia catalogue era. In Europe, the decline in interest in science amongst this age group is a concern as it forecasts a deficit of skilled professionals in the decades ahead. Outreach activities which bring the real experience of science to students play a role in efforts to reverse the declining number of scientists. An example of this is exemplified by the proefstuderen programme at Leiden University. Pre-university students are invited to attend a half day of lectures with the aim of giving them a sense of what is involved in university study. Anthony Brown (Leiden Observatory) uses material from the Hipparcos and Gaia missions in customized lectures. Descriptions of the missions, explanations of the science, and problem-solving exercises using real data are employed to expose the students to realistic scientific work. The students have the chance to be scientists for a day and at the same time they learn about the mission.

\subsection{A Gaia presence in every European planetarium}

More than 100 million visits to planetaria are registered each year (Petersen, 2005). These visitors are the "motivated, interested public" - a key audience for outreach professionals. Planetaria shows are delivered by talented presenters, planetarians, whose professional role is that of astronomy information disseminator. These are the people who decide what is relevant, interesting and appropriate for their audiences. Much of the material produced by outreach offices on behalf of science missions can be used by planetarians for their theatre presentations (Petersen, 2005). Press releases, images, animations, and background information may influence the content of live or pre-recorded planetarium shows. Ensuring, as a minimum, that outreach material on Gaia is made available to every planetarium in Europe will facilitate the inclusion of the mission in some of the aweinspiring and enthralling shows that are created. Forging links with local planetarians, and making oneself available to discuss the relevance of recent discoveries could ensure that a significant fraction of visitors to European planetaria are aware of the Gaia mission.

\subsection{The virtual world of Gaia}

Since its inception the internet has evolved from being a means of information exchange to one facilitating the interaction of web users and promoting aspects of social networking. One of the growth areas of the internet is that of the virtual world, for example, Second Life (http://secondlife.com). Outreach professionals have been quick to explore this new outlet: several museums have a virtual presence there, and a number of educational institutions are already running virtual classrooms. Although the number of users is presently small (a few million members) virtual worlds are expected to become a mainstay of internet interaction in the future. Outreach within a virtual world could take many forms: a virtual astronomer interacting with other avatars and giving night-sky tours, the virtual planetarium in which trips into the Gaia 3-d galaxy are one of the featured shows, virtual astronomy courses, ... the possibilities are vast. Those interested in outreach for Gaia should pay attention to developments in the virtual online world and be ready to take up the challenge offered by this new forum for communication.

\section{References}

Petersen, C. C., 2005, The Unique role of the planetarium/ science centre in science, Communicating Astronomy with the Public 2005

Fosbury, B., 2005, Difficult Concepts, Communicating Astronomy with the Public 2005 